Cómo citar este artículo: Rincón Flórez, J. F.; Fonseca Becerra, J. E. \& Carvajal Medina, R. J. (2015, julio-diciembre). Cálculo de parámetros termodinámicos para explosivos militares. Aplicación de fundamentos termodinámicos y propiedades de los explosivos militares. Rev. Cient. Gen. José María Córdova 13(16), 221-239

\title{
Cálculo de parámetros termodinámicos para explosivos militares. Aplicación de fundamentos termodinámicos y propiedades de los explosivos militares"
}

Recibido: 15 de marzo de 2015 - Aceptado: 21 de mayo de 2015.

Calculation of Thermodynamic Parameters for Military Explosives. Application of Thermodynamic Principles and Properties of Military Explosives

Calcul des paramètres thermodynamiques pour des explosifs militaires. Application des principes et des propriétés de la thermodynamique des explosifs militaires

Cálculo de parâmetros termodinâmicos para explosivos militares. Aplicaçáo dos princípios e das propriedades termodinâmicas de explosivos militares

John Fernando Rincón Flórez a

Jorge Enrique Fonseca Becerra ${ }^{b}$

Ricardo de Jesús Carvajal Medina ${ }^{c}$

*Artículo de investigación. Línea: "Uso de explosivos en Ingeniería Civil y Militar”, Especialización en Técnicas de Voladura en Obras de Ingeniería Civil y Militar, Escuela de Ingenieros Militares (Esing), Bogotá, Colombia.

a Ingeniero industrial, especialista tecnólogo en explosivos, Esing. Comentarios a: taless07@yahoo.es

b Químico, especialista tecnólogo en explosivos, esing. Comentarios a: jefonsecab@yahoo.com

c Magister en Educación, PhD (c) en Gerencia y Política Educativa; Asesor trabajos de grado de Escuela de Ingenieros Militares. Comentarios a: r.carvajal@esing.edu.co 
Resumen. Se realizan los cálculos de los parámetros termodinámicos de explosivos militares conocidos y utilizados con mayor regularidad, mostrando paso a paso como se realizan y tabulando los resultados, para lograr una comparación entre ellos. La estimación teórica de los parámetros termodinámicos permite considerar qué tipo de explosivo utilizar con más efectividad. Con los resultados obtenidos al realizar el cálculo de los parámetros termodinámicos, se puede hacer una formulación real que coincida con los datos teóricos ideales.

Palabras clave: explosivo, velocidad de detonación, brisance, entalpía ${ }^{(i)}$, presión de detonación, potencia absoluta, potencia relativa.

Abstract. Calculations of thermodynamic parameters of military explosives are made known and used more regularly, showing step by step how are realized and tabulating the results, to make a comparison between them. Theoretical estimation of thermodynamic parameters for considering that type of explosive used more effectively. With the results obtained in the calculation of the thermodynamic parameters, you can get to make a real formulation data match the theoretical ideal.

Keywords: absolute power, brisance, detonation speed, enthalpy, explosive, pressure detonation, relative power.

Résumé. Les calculs des paramètres thermodynamiques d'explosifs militaires soient connues et utilisées plus régulièrement, montrant étape par étape effectuée et la tabulation des résultats, de réaliser une comparaison entre eux. L'estimation théorique des paramètres thermodynamiques de considérer que type d'explosif utilisé plus efficacement. Avec les résultats obtenus dans le calcul des paramètres thermodynamiques, vous pouvez arriver à faire un réel des données de formulation correspondent à l'idéal théorique.

Mots-clés: brisance, enthalpie, explosive, pouvoir absolu, pression de détonation, puissance relative, vitesse de détonation.

Resumo. Cálculos dos parâmetros termodinâmicos de explosivos militares são feitas conhecido e utilizado com mais regularidade, mostrando passo a passo como foi executado e tabulação dos resultados, para assegurar uma comparação entre eles. A estimativa teórica de parâmetros termodinâmicos para considerar que tipo de explosivo utilizado de forma mais eficaz. Com os resultados obtidos no cálculo dos parâmetros termodinâmicos, você pode começar a fazer um conjunto de dados reais de formulação corresponde ao ideal teórico.

Palavras-chave: capacidade de detonação, entalpía, explosivo, poder absoluto, poder relativo, pressão de detonação, velocidade de detonação.

\section{Introducción}

En el contexto del conflicto interno colombiano, los ingenieros militares han trabajado por el bienestar de la comunidad. En este sentido, una de las misiones de los ingenieros militares es su vocación por el desarrollo de obras generales de ingeniería, verticales y horizontales, tales como: carreteras, perforación de pozos, construcción de edificaciones, entre otras obras de infraestructura que tienen gran impacto social, sobre todo en zonas apartadas del país afectadas por el actual

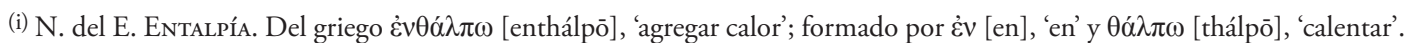
Magnitud termodinámica, simbolizada con la letra griega etha en mayúscula $(\mathrm{H})$, cuya variación expresa una medida de la cantidad de energía absorbida o cedida por un sistema termodinámico, es decir, la cantidad de energía que un sistema intercambia con su entorno. 
conflicto armado. En un escenario de posconflicto, los ingenieros militares también serán determinantes para desarrollar las obras de infraestructura en dichas regiones de difícil acceso, lo cual redundará en beneficio del desarrollo del país en materia de obras de ingeniería, y en la construcción de de mejores condiciones de vida para las comunidades más apartadas.

Esa vocación y ese espíritu que hoy impulsan el desempeño profesional de los ingenieros militares han sido el legado del Coronel de Ingenieros Francisco José de Caldas, más comúnmente conocido como el Sabio Caldas, no solo por sus aportes significativos en la fabricación de piezas de artillería, sino también por sus estudios matemáticos y científicos en ingeniería militar, y especialmente por concebir un estatuto ético que ha servido para impulsar la fuerza moral de toda persona que opte por la profesión de las armas, en su Discurso preliminar (edición facsimilar en Paredes, 2010, pp. 312-340) ${ }^{(i i)}$.

En el Discurso preliminar del Sabio Caldas, en efecto, se fijan los principales valores y consignas del Arma de Ingenieros Militares en Colombia, que ha dejado una indeleble impronta en el derrotero de doscientos años de vida republicana, construyendo país y constituyéndose por sus méritos en el Arma de la Paz.

La Advertencia, o Introducción al Discurso, ha sido un horizonte axiológico de nuestra Arma de Ingenieros:

Nada es mas util en una profesion que el conocimiento de las obligaciones en que el hombre se ha constituido, abrazandolo; y ninguno puede hacer sólidos progresos ignorando sus deberes, y las virtudes que deben adornarlo. El Cuerpo de Ingenieros poseido de esta verdad ha creido hacer un servicio a todos los militares dando a la prensa el Discurso preliminar que se le ha leído al principio de su curso.

Manuel María Viller Calderón, editor, Advertencia al Discurso preliminar (Caldas, 1815, folio 2), ortografía de la época e itálicas en el original.

Con esto en mente, una de las líneas de investigación de la Escuela de Ingenieros Militares es el uso de explosivos en ingeniería cívil y militar. En esta línea, se han desarrollado investigaciones que permiten un conocimiento más especializado de los explosivos militares y comerciales, con el propósito de hacer un uso más adecuado y con un mejor rendimiento en las obras de infraestructura tanto civiles como militares.

(ii) N. del E. En esta obra, destinada a celebrar los 200 años de historia de los ingenieros militares, se rescatan los momentos, personajes, procesos y logros más importantes del Arma de Ingenieros durante dos siglos de vida republicana, a la vez que se esbozan su esencia, sus valores y su labor, fundamentales para alcanzar la paz e impulsar el desarrollo del país. Cabe destacar que allí se encuentra la primera reproducción que se hizo del discurso, que en esta edición se reproduce en la sección ReseŃas, gracias a los buenos oficios de la Escuela de Ingenieros para su máxima difusión en el mundo académico. El Discurso preliminar fue publicado en el año de 1815 en Medellín, en la Imprenta del Gobierno, a expensas del Cuerpo de Ingenieros, por el ciudadano Manuel María Viller Calderón, en el tercer año de la independencia de Colombia. El discurso fue leído por el Sabio Caldas en octubre de 1814, en la Escuela Militar de Rionegro, o primera Escuela de Ingenieros que se estableció en el país, en la entonces República de Antioquia, que existió entre los ańos 1813 y 1816 en el territorio que hoy corresponde al departamento de Antioquia, época en la cual ingresó el alumno José María Córdova, héroe epónimo de nuestra Escuela Militar de Cadetes, según Resolución 0076 del 24 de agosto 1998 del Ejército Nacional. El primer número de nuestra Revista comienza con la publicación del Discurso preliminar, adaptado a la ortografía moderna, con subtítulos del Editor, que tienen por objeto facilitar su lectura, según los tópicos doctrinales del texto. Cf. Rev. Cient. Gen. José María Córdova 1(1), pp. 3-12. 
Para responder a ese desafío de investigación, la Escuela de Ingenieros Militares es la única institución del país que cuenta con programas de especialización en el área de técnicas de explosivos y voladuras, como la Especialización en técnicas de voladura en obras de ingeniería civil y militar, debidamente avalada por parte del Ministerio de Educación Nacional, y con cerca de 150 egresados.

La producción de explosivos en Colombia es exclusiva del sector Defensa, especialmente de la industria militar. El uso y la manipulación está a cargo de grupos especializados, que para el caso del ejército se denominan grupos MARTE (Manejo de Artefactos Explosivos), grupos EXDE (Explosivos y Demoliciones), grupos DELTA y grupos GECOES; todos especializados en la lucha contra Artefactos Explosivos Improvisados (AEI), con diferentes niveles de conocimiento y menor o mayor radio de acción. El conocimiento técnico que se ha desarrollado por parte de estos grupos especializados ha permitido combatir a los grupos terroristas, especialmente en el tema de las minas antipersonales. Sin embargo, este conocimiento también ha permitido realizar investigaciones sobre desarrollos tecnológicos en materia de explosivos cuyos resultados y discusión se presentan en el presente trabajo, con el propósito de profundizar en el conocimiento de la naturaleza misma de los explosivos, para controlar mejor el uso de explosivos en el desarrollo de obras de ingeniería civil y militar.

Con esto en mente, el artículo discute los cálculos paso a paso para los diferentes explosivos analizados, de tal forma que el especialista en explosivos pueda saber cómo se obtienen los resultados y pueda analizar y sacar sus propias conclusiones con respecto al desempeńo de estos materiales en una voladura determinada.

Al final del trabajo se encuentra resultados teóricos y experimentales de tres explosivos (Composición B con formulación diferente entre sí, Pentolita y PTX-II), los cuales podrán usarse como protocolo o guía para la experimentación y determinación de parámetros termodinámicos. Esto permitirá realizar una formulación de un explosivo determinado para uso militar o comercial, luego de comparar los resultados obtenidos de parámetros termodinámicos con el desempeńo del explosivo elaborado.

Esta investigación se realizó en la Escuela de Ingenieros Militares, dentro de la línea uso de explosivos en ingeniería civil y militar.

\section{Metodología}

Para la realización de los cálculos termodinámicos de explosivos militares, se utilizaron las fórmulas dadas por diferentes autores y encontradas en diferentes manuales (Cf. López, 1988; Exsa, 2008; Carrillo, 2003).

\section{Balance de oxígeno, $\mathrm{BO}$}

Se define como la cantidad de oxígeno presente en un explosivo (Meyer, Kohler \& Homburg, 2007 , p. 20). La determinación de este parámetro químico se halla midiendo previamente las constantes de oxígeno de cada una de las sustancias químicas presentes o no en una mezcla de materiales que compone un explosivo. 


\section{Cálculo de la constante de oxígeno}

Es la cantidad de oxígeno $\left(\mathrm{Cte}\right.$. de $\mathrm{O}_{2}$ ) necesario para la reacción completa de los reactantes en una detonación. Esta se calcula por medio de la ecuación (1):

$$
\text { Cte. } 02=\frac{\text { moles de } 02 \times \text { peso molecular del } 02(\text { en gramos })}{\text { moles del compuesto } \times \text { peso molecular del compuesto }(\text { en gramos })}
$$

El valor de la Cte. de $\mathrm{O}_{2}$ en la ecuación (1) es positivo, cuando en la reacción el compuesto cede o libera $\mathrm{O}_{2}$, y es negativo, porque necesita o recibe cuando el $\mathrm{O}_{2}$ para su reacción completa.

Los explosivos generalmente son mezclas de sustancias químicas de tipo oxidante y combustible. El balance de oxígeno (BO) se expresa generalmente como un porcentaje; y se calcula generalmente multiplicando el porcentaje presente de cada una de las sustancia químicas presentes en el explosivo por su respectiva constante de oxígeno; y sus resultados se suman.

$\mathrm{BO}($ Explosivo $)=\left(\%\right.$ sustancia $1 \times$ Cte. de $\mathrm{O}_{2}$ sustancia 1$)+\left(\%\right.$ Sustancia $2 \times$ Cte. de $\mathrm{O}_{2}$ sustancia 2)

En el caso de deficiencia de oxígeno, el balance se da con signo negativo. En muchos explosivos la sensibilidad, la potencia y el poder rompedor aumentan conforme lo hace el balance de oxígeno, hasta alcanzar un máximo en el punto de equilibrio.

En los explosivos con balance de oxígeno positivo, el oxígeno disponible se combina con los átomos de carbono para formar $\mathrm{CO}_{2} \mathrm{y}$ óxidos de nitrógeno, algunos de color rojo. Los humos rojos indican una cantidad de combustible insuficiente en la reacción, que puede ser debida a una mezcla, segregación o pérdida de combustible.

Los explosivos con balance de oxígeno negativo forman óxidos incompletos, en particular $\mathrm{CO}$, que es venenoso e incoloro. Los gases nitrosos se reducen mucho, por lo que en bastantes casos los explosivos se formulan con un pequeño balance de oxígeno negativo.

\section{Presión de detonación}

Es el resultado del movimiento de la onda de choque a través del explosivo (Cf. López, 1988; Exsa, 2008; Carrillo, 2003, p.11).

La presión de detonación está relacionada con la densidad del explosivo y la velocidad de detonación.

Es un indicador significativo de la capacidad de fragmentación que posee un explosivo.

La presión de detonación está dada por la fórmula de la ecuación (2):

$$
P D=\mathrm{K} * \mathrm{DENS} \exp * \mathrm{VOD}^{2} \exp
$$




\section{Calor de formación (entalpía)}

El valor de la entalpía de formación estándar, definen los calores de formación (entalpías) en kilocalorías por mol de las sustancias participantes en los componentes del explosivo (Cf. López, 1988; Exsa, 2008; Carrillo, 2003, cap.1).

En un compuesto, indica la estabilidad del mismo, puesto que a mayor calor desprendido en su formación $\left(\Delta \mathrm{H}_{\mathrm{f}}{ }^{\circ}<0\right)$ mayor será su estabilidad, de modo que se necesitará más calor para su descomposición.

Es, por definición, la variación de entalpía que acompaña a la formación de un mol de compuesto, a partir de los elementos que la componen a una presión y temperatura determinadas.

\section{Calor de explosión}

Es el calor generado y liberado por el proceso de reacción de un explosivo al ser activado (Cf. López, 1988; Exsa, 2008; Carrillo, 2003, p.13).

Cuando se produce una explosión a presión constante, ejerciendo únicamente un trabajo de expansión o compresión, la primera ley de la termodinámica (véase ecuación 3) establece que:

$$
Q c=\Delta(\mathrm{Uc}+(\mathrm{P} \times \mathrm{V})
$$

Donde:

Qc: calor liberado por la explosión.

Uc: energía interna del explosivo.

P: presión.

$\mathrm{V}$ : volumen.

Así el calor de explosión a presión constante es igual al cambio de entalpía y puede estimarse estableciéndose el balance térmico de la reacción, multiplicando los calores de formación de los productos finales por el número de moles que se forma de cada uno, sumándolos para restar a continuación el calor de formación del explosivo (véase ecuación 4).

$\mathrm{Hp}($ explosivo $)=\mathrm{Hp}($ productos $)-\mathrm{Hp}$ (explosivo)

O también dicho de otro modo:

$$
Q e=Q p-Q r
$$

Donde:

Qe: calor total de explosión liberado.

Qp: calor total de formación de los productos finales resultantes.

Qr: calor total de formación de los productos componentes.

$\mathrm{Qp}=\#$ moles produc $\mathrm{C} \times \Delta \mathrm{H}_{\mathrm{f}}^{\mathbf{o}}$ produc $\mathrm{C}+\#$ moles produc $\mathrm{D} \times \Delta \mathrm{H}_{\mathrm{f}}$ ) produc $\mathrm{D}$ 
$\mathrm{Qp}=\Sigma$ (\#moles productos finales resultantes $\times \Delta \mathrm{H}_{\mathrm{f}}$ o productos finales resultantes)

$\mathrm{Qr}=\#$ moles pr A $x \Delta \mathrm{H}_{\mathrm{f}}{ }^{\circ}$ pr A + \#moles pr B x $\Delta \mathrm{H}_{\mathrm{f}}$ opr B

$\mathrm{Qr}=\Sigma$ (\#moles productos componentes $\mathrm{x} \Delta \mathrm{H}_{\mathrm{f}} \mathbf{o}$ productos componentes)

El peso molecular (PM) del explosivo, según la ecuación 5, es:

$$
P M=(\# \text { moles } A \text { x pm } A)+(\# \text { moles } B \times \text { pm } B)
$$

\section{Cálculo de la potencia relativa}

La potencia relativa por peso (Cf. López, 1988; Exsa, 2008; Carrillo, 2003), conocida por sus iniciales en inglés, RWS (Pernia-Llera et al. 1989) es el resultado de dividir (véase ecuación 6) la potencia absoluta por peso, PAP, (energía por unidad de masa) en cal/g del explosivo en cuestión, entre la PAP del explosivo patrón.

$$
R W S=\frac{\text { PAP exp a calcular }}{\text { PAP exp patron }}
$$

Potencia relativa por volumen (véase ecuación 7), conocida por sus iniciales en inglés RBS (PerniaLlera et al. 1989), es el resultado de dividir la potencia absoluta por volumen, PAV, (energía por unidad de volumen) del explosivo en cuestión, entre la PAV del explosivo patrón, para lo que debemos tener en cuenta que la PAV de un explosivo es igual a su PAP en cal/g, por su densidad Pe, en $\mathrm{g} / \mathrm{cm}^{3}$.

$$
R B S=\frac{\text { PAP } \times \text { De }}{\text { PAPp } \times \text { Dep }} \quad \text { ó } \quad R B S=\frac{\mathrm{PAV}}{\mathrm{PAVp}}
$$

Donde:

PAP = Potencia absoluta en peso del explosivo en cuestión, cal/g

De $=$ Densidad del explosivo en cuestión, $\mathrm{g} / \mathrm{cm}^{3}$

PAPp y Dep. = se refieren al explosivo patrón.

PAV = Potencia absoluta en volumen del explosivo en cuestión.

$\mathrm{PAVp}=$ potencia absoluta en volumen del explosivo patrón.

La potencia relativa es conocida en algunos textos como FER (factor de efectividad relativa) y por lo general es calculado con los valores del TNT.

La potencia relativa es utilizada en volumen cuando se compara con el volumen de gases producidos por el explosivo y su poder rompedor.

Para realizar los anteriores cálculos tanto termodinámicos como propiedades de explosivos tomamos como ejemplo la Pentolita, aclarando de antemano que todos estos parámetros se rea- 
lizan de la misma forma para cualquier explosivo comercial o militar (Department of the Army Technical Manual, 1984; Sociedad Española de la Dinamita, 1928).

Lo primero es realizar los cálculos de cada uno de los componentes refiriéndonos a la PETN y TNT para la mezclas explosiva llamada Pentolita.

Aquí solo se darán los necesarios para el cálculo en el compuesto.

\section{PETN}

Fórmula química: $\mathrm{C}_{5} \mathrm{H}_{8} \mathrm{~N}_{4} \mathrm{O}_{12}$

Densidad: $1,7 \mathrm{~g} / \mathrm{cm}^{3}$

Velocidad: detonación $8400 \mathrm{~m} / \mathrm{s}$.

\section{Cálculo de la constante de oxígeno}

Cantidad de oxígeno necesaria para su reacción completa (véase ecuaciones 8, 9 y 10):

$$
\mathrm{C}_{5} \mathrm{H}_{8} \mathrm{~N}_{4} \mathrm{O}_{12}+\mathrm{O}_{2}====>5 \mathrm{CO}_{2}+4 \mathrm{H}_{2} \mathrm{O}+2 \mathrm{~N}_{2}
$$

Tabla 1. Peso molecular de la Pentrita.

\begin{tabular}{ccccc}
\hline & $\begin{array}{c}\text { Elemento } \\
\text { Quimico }\end{array}$ & $\begin{array}{c}\text { Numero de } \\
\text { atomos en el } \\
\text { compuesto }\end{array}$ & $\begin{array}{c}\text { Peso molecular } \\
\text { del elemento }\end{array}$ & $\begin{array}{c}\text { Peso molecular total } \\
\text { (multiplicar numero de } \\
\text { atomos por peso molecular) }\end{array}$ \\
\cline { 2 - 5 } & $\mathrm{C}$ & 5 & 12 & 60 \\
\hline $\mathrm{H}$ & 8 & 1 & 8 \\
\hline $\mathrm{N}$ & 4 & 14 & 56 \\
\hline
\end{tabular}

Fuente: elaboración propia, con fundamento en Sociedad Española de la Dinamita, 1928.

Cte. $\mathrm{O}_{2}=\frac{\text { moles de } 02 x \text { peso molecular del } 02 \text { (en gramos })}{\text { moles del compuesto } x \text { peso molecular del compuesto (en gramos) }}$

$$
\text { Cte. } \mathrm{O}_{2}=\frac{-2 \times 16 \mathrm{~g}}{1 \times 316 \mathrm{~g}} \Longrightarrow \text { Cte. } \mathrm{O}_{2}=\frac{-32}{316} \quad \text { Cte. } \mathrm{O}_{2}=-0,101
$$

$\mathrm{El}$ valor de la Cte. de $\mathrm{O}_{2}$ es negativo porque recibe $\mathrm{O}_{2}$. 


\section{Trinitrotolueno TNT}

Fórmula molecular: $\mathrm{C}_{6} \mathrm{H}_{2}\left(\mathrm{NO}_{2}\right)_{3} \mathrm{CH}_{3}$.

Densidad: $1,6 \mathrm{~g} / \mathrm{cm}^{3}$

Velocidad de detonación: $6900 \mathrm{~m} / \mathrm{s}$

\section{Cálculo de la constante de oxígeno}

Cantidad de oxígeno necesaria para su reacción completa (véase ecuaciones 11, 12 y 13):

$$
2 \mathrm{C}_{7} \mathrm{H}_{5} \mathrm{~N}_{3} \mathrm{O}_{6}+10,5 \mathrm{O}_{2}====>14 \mathrm{CO}_{2}+5 \mathrm{H}_{2} \mathrm{O}+3 \mathrm{~N}_{2}
$$

Tabla 2. Peso molecular del TNT

\begin{tabular}{|c|c|c|c|c|}
\hline \multirow{5}{*}{ 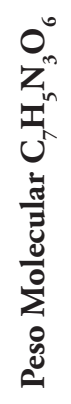 } & $\begin{array}{l}\text { Elemento } \\
\text { Quimico }\end{array}$ & $\begin{array}{l}\text { Numero de ato- } \\
\text { mos en el com- } \\
\text { puesto }\end{array}$ & $\begin{array}{l}\text { Peso molecular } \\
\text { del elemento }\end{array}$ & $\begin{array}{l}\text { Peso molecular total ( multi- } \\
\text { plicar numero de atomos por } \\
\text { peso molecular) }\end{array}$ \\
\hline & $\mathrm{C}$ & 7 & 12 & 84 \\
\hline & $\mathrm{H}$ & 5 & 1 & 5 \\
\hline & $\mathrm{N}$ & 3 & 14 & 42 \\
\hline & $\mathrm{O}$ & 6 & 16 & 96 \\
\hline \multicolumn{4}{|c|}{ TOTAL (EN g) } & 227 \\
\hline
\end{tabular}

Fuente: elaboración propia, con fundamento en Sociedad Espańola de la Dinamita, 1928.

$$
\text { Cte. } \mathrm{O}_{2}=\frac{\text { moles de } 02 x \text { peso molecular del } 02 \text { (en gramos })}{\text { moles del compuesto } x \text { peso molecular del compuesto (en gramos) }}
$$

Cte. $\mathrm{O}_{2}=\frac{-21 \times 16 \mathrm{~g}}{2 \times 227 \mathrm{~g}} \Longrightarrow$ Cte. $\mathrm{O}_{2}=\frac{-336}{454} \quad$ Cte. $\mathrm{O}_{2}=-0,740$

El valor de la Cte. de $\mathrm{O}_{2}$ es negativo porque recibe $\mathrm{O}_{2}$. 


\section{Pentolita 50/50}

Velocidad de detonación: $6700 \mathrm{~m} / \mathrm{s}$

Densidad: $1,6 \mathrm{~g} / \mathrm{cm}^{3}$

PETN $\left\{\mathrm{C}_{5} \mathrm{H}_{8} \mathrm{~N}_{4} \mathrm{O}_{12}\right\} \quad 50 \%$.

TNT $\left\{\mathrm{C}_{7} \mathrm{H}_{5} \mathrm{~N}_{3} \mathrm{O}_{6}\right\} \quad 50 \%$.

\section{Reacción química ideal}

PETN + TNT + Oxígeno ====> agua + nitrógeno libre + dióxido de carbono

$$
\mathrm{C}_{5} \mathrm{H}_{8} \mathrm{~N}_{4} \mathrm{O}_{12}+2 \mathrm{C}_{7} \mathrm{H}_{5} \mathrm{~N}_{3} \mathrm{O}_{6}+11,5 \mathrm{O}_{2}===>9 \mathrm{H}_{2} \mathrm{O}+5 \mathrm{~N}_{2}+19 \mathrm{CO}_{2}
$$

\section{Balance de oxígeno}

BO PENTOLITA $=(\%$ PENT $\mathrm{x}$ cte oxig PENT $)+(\%$ TNT $\mathrm{x}$ cte oxig TNT $)$

Mezcla 50/50

$\mathrm{BO}$ pentolita $=(50 \% \mathrm{X}-0,101)+(50 \% \mathrm{X}-0,74)$

$\mathrm{BO}$ Pentolita $=(0,5 \mathrm{X}-0,101)+(0,5 \mathrm{X}-0,74)$

$\mathrm{BO}$ PENTOLITA $=(0,0505)+(0,37)$

BO PENTOLITA $=-0,4205$

\section{Presión de detonación}

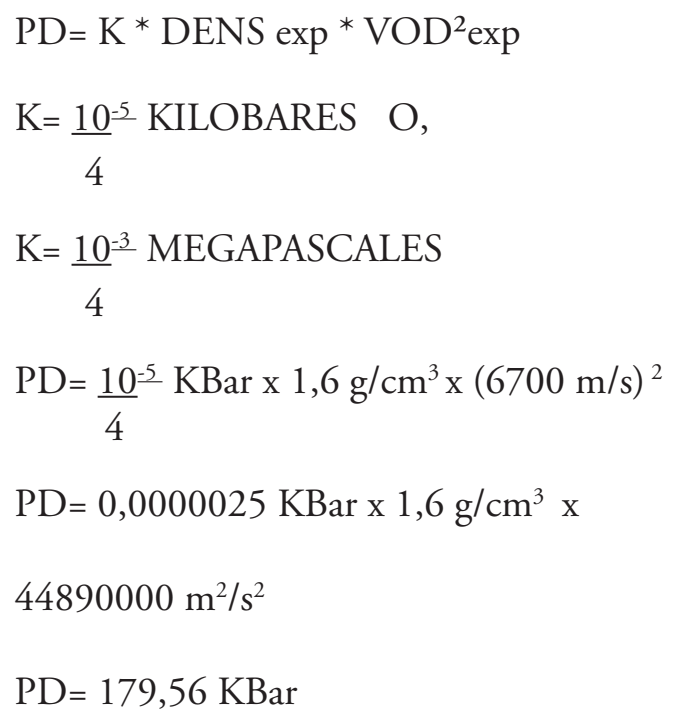


$\mathrm{PD}=\underline{10^{-3}}-\mathrm{MPa} \times 1,6 \mathrm{~g} / \mathrm{cm}^{3} \times(6700 \mathrm{~m} / \mathrm{s})^{2}$

$\mathrm{PD}=0,00025 \mathrm{MPa} \times 1,6 \mathrm{~g} / \mathrm{cm}^{3} \mathrm{x}$

$44890000 \mathrm{~m}^{2} / \mathrm{s}^{2}$

$\mathrm{PD}=17956 \mathrm{MPa}$

\section{Potencia absoluta en peso o calor de explosión}

Tabla 3. Calor de formación y peso molecular de la Pentolita

\begin{tabular}{ccc}
\hline Producto & $\begin{array}{c}\text { Calor de formación } \\
(\mathbf{K c a l} / \mathbf{m o l})\end{array}$ & $\begin{array}{c}\text { Peso molecular } \\
(\mathbf{G})\end{array}$ \\
\hline PETN $\left(\mathrm{C}_{5} \mathrm{H}_{8} \mathrm{~N}_{4} \mathrm{O}_{12}\right)$ & $+462,94$ & 316 \\
\hline $\begin{array}{c}\text { Trinitrotolueno }(\mathrm{TNT}) \\
\left(\mathrm{C}_{7} \mathrm{H}_{5} \mathrm{~N}_{3} \mathrm{O}_{6}\right)\end{array}$ & $-16,03$ & 227 \\
\hline Oxigeno $\left(\mathrm{O}_{2}\right)$ & 0 & 32 \\
\hline Dióxido de carbono $(\mathrm{CO} 2)$ & $-94,1$ & 44,0 \\
\hline Agua $\left(\mathrm{H}_{2} \mathrm{O}\right)$ & $-57,8$ & 18,0 \\
\hline Nitrógeno $(\mathrm{N})$ & 0 & 42 \\
\hline
\end{tabular}

Fuente: elaboración propia, con fundamento en Meyer, Kohler \& Homburg, 2007.

$$
\begin{aligned}
& \begin{array}{l}
\mathrm{C}_{5} \mathrm{H}_{8} \mathrm{~N}_{4} \mathrm{O}_{12}+2 \mathrm{C}_{7} \mathrm{H}_{5} \mathrm{~N}_{3} \mathrm{O}_{6}+11,5 \mathrm{O}_{2}==== \\
(\text { Explosivo })
\end{array} \\
& (\mathrm{Cp})=1(462,94)+2(-16,03)+11,5(0) \\
& (\text { Productos de reacción) }
\end{aligned}
$$$$
\text { (Productos de reacción) }
$$

\section{Luego}

$\mathrm{Qe}=\mathrm{Qp}-\mathrm{Qr}$; calor de explosión,

$\mathrm{Qe}=430,88 \mathrm{kcal} / \mathrm{mol}-(-2308,1) \mathrm{kcal} / \mathrm{mol}=2738,98 \mathrm{kcal} / \mathrm{mol}$ 


$$
\begin{aligned}
& \mathrm{PM}=1(316)+2(227)+11,5(32) \\
& \mathrm{PM}=316+454+368=1138 \mathrm{~g} \\
& \mathrm{QKp}=\underline{\mathrm{Qe}} \\
& \mathrm{PM}
\end{aligned}
$$

$\mathrm{QKp}=2308,1 \underline{\mathrm{kcal}} \times \underline{1 \mathrm{~mol}} \times \underline{1000 \mathrm{~g}}$

$$
\text { mol } 1138 \mathrm{~g} \quad 1 \mathrm{~kg}
$$

$\mathrm{Qkp}=2028,2 \mathrm{kcal} / \mathrm{kg} \quad, \mathrm{O}$,

$$
\mathrm{QKp}=2308,1 \underline{\mathrm{kcal}} \times \frac{1 \mathrm{~mol}}{1138 \mathrm{~g}} \times \frac{1000 \mathrm{~g}}{1 \mathrm{kcal}}
$$

$\mathrm{Qkp}=2028,2 \mathrm{cal} / \mathrm{g}$

$\mathrm{Qmv}=\mathrm{Qe}+0,58 \times \mathrm{Npg}$

Donde:

Npg: número de moles de productos gaseosos.

$\mathrm{Qmv}=2308,1+(33 \times 0,58)=2327,24 \mathrm{kcal} / \mathrm{mol}$

$\mathrm{Qkv}=\mathrm{Qmv} \times \frac{1 \mathrm{~mol}}{\mathrm{PM}} \times \frac{1000 \mathrm{~g}}{1 \mathrm{~kg}}$

$\mathrm{Qkv}=2327,24 \underline{\mathrm{kcal}} \times \underline{1 \mathrm{~mol}} \times \underline{1000 \mathrm{~g}}$

$$
\text { mol } \quad 1138 \mathrm{~g} \quad 1 \mathrm{~kg}
$$

$\mathrm{Qkv}=2045,02 \mathrm{kcal} / \mathrm{kg} \quad$ ó

$$
\mathrm{Qkv}=2327,24 \underline{\mathrm{kcal}} \times \frac{1 \mathrm{~mol}}{1138 \mathrm{~g}} \times \frac{1000 \mathrm{cal}}{1 \mathrm{kcal}}
$$

$\mathrm{Qkv}=2045,02 \mathrm{cal} / \mathrm{g}$

$$
V \exp =\frac{P M}{D e}
$$




$$
\begin{aligned}
& \text { Vexp }=\frac{1138 \mathrm{~g}}{1,6 \mathrm{~g} / \mathrm{cm}^{3}}=711,25 \mathrm{~cm}^{3} \\
& \mathrm{Qkv}=\mathrm{Qmv} \times \frac{1 \mathrm{~mol}}{\operatorname{Vexp}} \times \frac{1000 \mathrm{cal}}{1 \mathrm{kcal}} \\
& \mathrm{Qkv}=2327,24 \underline{\mathrm{kcal}} \times \frac{1 \mathrm{~mol}}{\mathrm{~mol}} \times \frac{1000 \mathrm{cal}}{711,25 \mathrm{~cm}^{3} 1 \mathrm{kcal}} \\
& =3272,04 \mathrm{cal} / \mathrm{cm}^{3}
\end{aligned}
$$

\section{Factor de potencia}

$$
\begin{aligned}
& \mathrm{FP}=2028,2 \mathrm{cal} / \mathrm{g} \times 6700 \mathrm{~m} / \mathrm{s} \times 1,6 \mathrm{~g} / \mathrm{cm}^{3} \\
& \mathrm{FP}=\left(21742304 \mathrm{cal}^{*} \mathrm{~m} / \mathrm{s}^{*} \mathrm{~cm}^{3}\right)(1 \mathrm{Joul} / 0,2388 \mathrm{cal})\left(1000000 \mathrm{~cm}^{3} / 1 \mathrm{~m}^{3}\right) \\
& \mathrm{FP}=9,104 \times 10^{13} \mathrm{~Pa}^{*} \mathrm{~m} / \mathrm{s}
\end{aligned}
$$

\section{Volumen de explosión ideal}

El volumen o mol de la molécula-gramo de cualquier gas, en condiciones normales es 22,4 litros.

$$
\mathrm{C}_{5} \mathrm{H}_{8} \mathrm{~N}_{4} \mathrm{O}_{12}+2 \mathrm{C}_{7} \mathrm{H}_{5} \mathrm{~N}_{3} \mathrm{O}_{6}+11,5 \mathrm{O}_{2}====>19 \mathrm{CO}_{2}+9 \mathrm{H}_{2} \mathrm{O}+5 \mathrm{~N}_{2}
$$

La explosión de 1 mol genera:

N.mol productos gaseosos $=19+9+5=33$

N.mol productos gaseosos $=33 / 3=11 \mathrm{~mol}$ productos gaseosos a $0^{\circ} \mathrm{C}$ y a presión atmosférica de una atmosfera.

Vol explosión $=11 \mathrm{~mol}$ x 22,4 litro $/ \mathrm{mol}=246,4$ litros

\section{Volumen de explosión ideal Cálculos de la potencia relativa o FER (factor de efectividad relativa)}

\section{Cálculos RWS}

PAP Pentolita: $2028,2 \mathrm{cal} / \mathrm{cm}^{3}$

$$
\text { RWS Pentolita }=\frac{2028,2 \mathrm{cal} / \mathrm{cm}^{3}}{2028,2 \mathrm{cal} / \mathrm{cm}^{3}}=1
$$


RWS Pentrita $=\underline{3324,94 \mathrm{cal} / \mathrm{cm}^{3}}=1,639$

$2028,2 \mathrm{cal} / \mathrm{cm}^{3}$

RWS TNT $=\underline{1992,84 \mathrm{cal} / \mathrm{cm}^{\underline{3}}}=0,983$

$2028,2 \mathrm{cal} / \mathrm{cm}^{3}$

\section{Cálculos RBS}

PAV Pentolita $=3272,04 \mathrm{cal} / \mathrm{cm}^{3}$

RBS Pentolita $=\underline{3272,04 \mathrm{cal} / \mathrm{cm}^{\underline{3}}}=1$

$3272,04 \mathrm{cal} / \mathrm{cm}^{3}$

RBS Pentrita $=5683,45 \mathrm{cal} / \mathrm{cm}^{3}=1,737$

$3272,04 \mathrm{cal} / \mathrm{cm}^{3}$

RBS TNT $=\underline{3214,38 \mathrm{cal} / \mathrm{cm}^{3}}=0,982$

$3272,04 \mathrm{cal} / \mathrm{cm}^{3}$

\section{Resultados}

Resultados similares a los obtenidos para explosivos simples (PETN, TNT) y mezclas de explosivos simples como la Pentolita fueron obtenidos en este trabajo para otros explosivos militares, determinándose la energía del explosivo como potencia absoluta en peso y/o potencia absoluta en volumen o potencia relativa en peso y/o potencia relativa en volumen. Otras características principales de estos materiales fueron determinados como; constante de oxígeno y/o balance de oxigeno de componentes del explosivo, presión de detonación, factores de potencia y volumen de explosión en cuanto a gases producidos.

A continuación se anexa tabla de resultados de energía y otras propiedades de los explosivos militares (ver tabla 4).

De otra parte se realizaron las estimaciones de energía y características de estos materiales en tres muestras de explosivos preparadas a nivel de laboratorio realizando la determinación de características fisicoquímicas cada uno de los explosivos puros y compuestos. Una de estas mezclas fue la composición B preparada en tres composiciones químicas diferentes y se realizaron otras dos como PTX-II y Pentolita.

Las tres composiciones formuladas para la composición B fueron: Composición B (Mezcla I: 60\% RDX-39\% TNT-1 \% Cera; Mezcla II: 60 \%TNT-39\%RDX-1\% Cera; Mezcla III: 49,5\%RDX-49,5\%TNT-1\%Cera); para la Pentolita(50\%TNT-50\%PETN) y para el PTX-II (44\%RDX-28\%TNT-28\%PETN).

A continuación se anexa los datos obtenidos de energía y otros parámetros de explosivos para estos materiales (Ver tabla 5): 


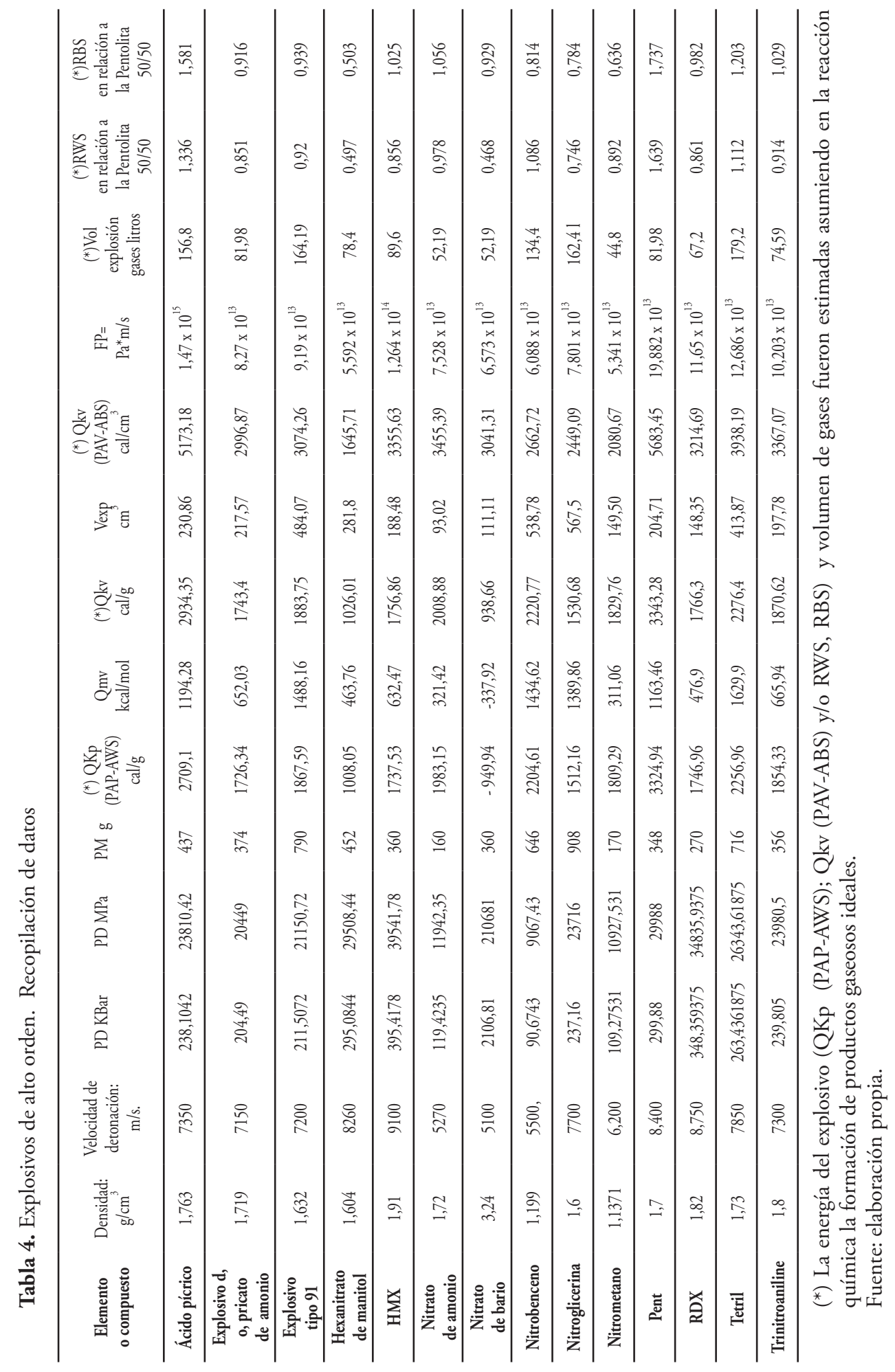




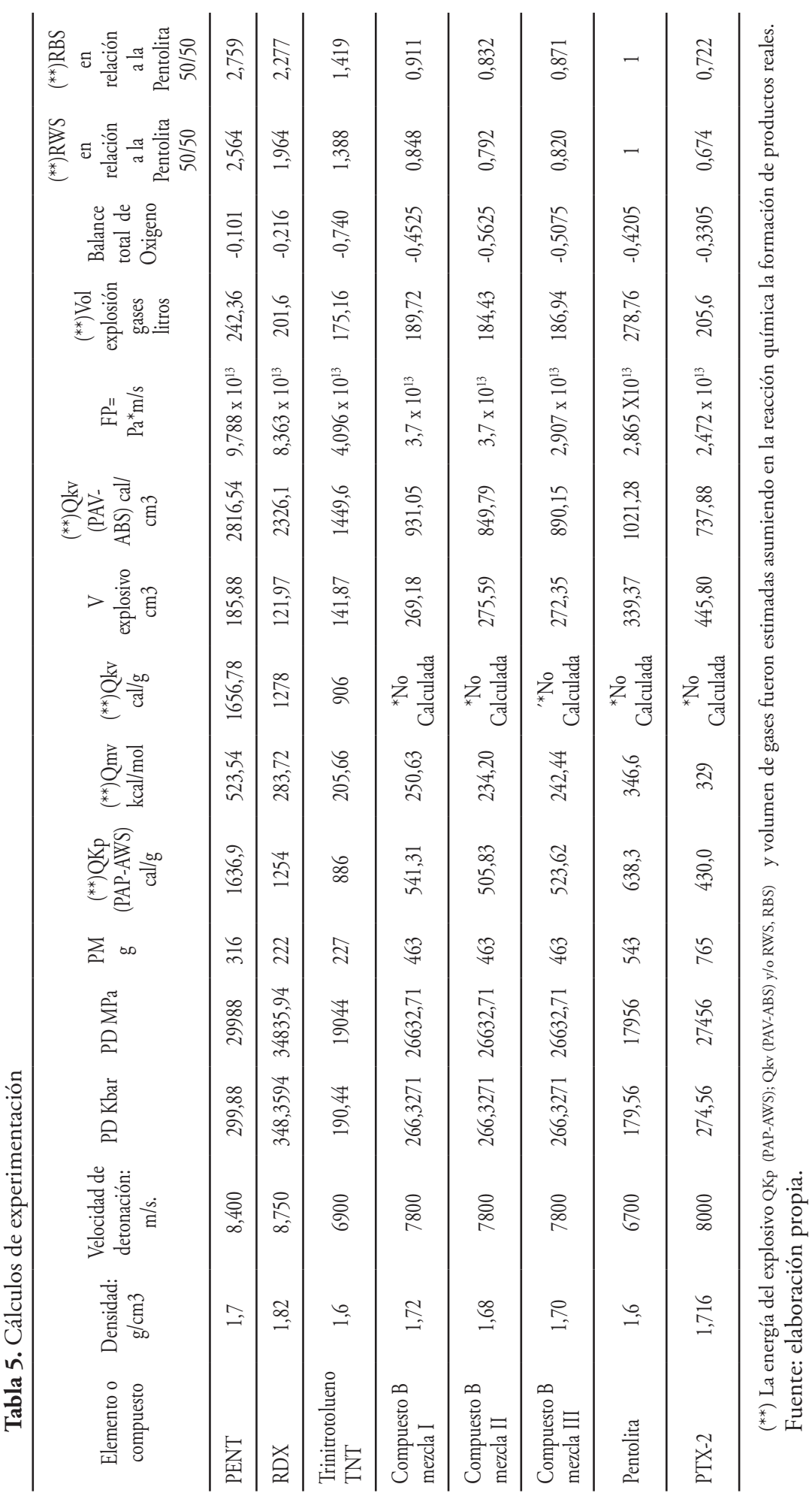


Se realizaron pruebas balísticas para verificar la sensibilidad al detonador, el poder rompedor (Brisance) por medio de la prueba de Hess y péndulo [2], [3], [5], [6]. Los resultados de estas pruebas se compararon de nuevo con el explosivo referencia que es la Pentolita y los resultados se tabularon y cuantificaron de forma individual. En la tabla 6 se muestran algunos de los resultados obtenidos en las pruebas balística

Tabla 6. Muestras y resultados de pruebas composición B Mezcla I a

\begin{tabular}{lccccc}
\hline $\begin{array}{c}\text { Número } \\
\text { muestra }\end{array}$ & Peso tubo cartón & $\begin{array}{c}\text { Peso neto } \\
\text { muestra }\end{array}$ & $\begin{array}{c}\text { Longitud } \\
\text { del cilindro }\end{array}$ & $\begin{array}{c}\text { Deformación } \\
\text { producida }\end{array}$ & $\begin{array}{c}\text { \%Deformacion } \\
\text { producida }\end{array}$ \\
\hline 1a HESS & 5,4012 & 39,485 & $50,2 \mathrm{~mm}$ & $39,42 \mathrm{~mm}$ & $21,48 \%$ \\
\hline 2b HESS & 5,2641 & 42,6637 & $50 \mathrm{~mm}$ & $39 \mathrm{~mm}$ & $22 \%$ \\
\hline 3c HESS & 5,2930 & 42,2319 & $50,6 \mathrm{~mm}$ & $39,22 \mathrm{~mm}$ & $22,5 \%$ \\
\hline
\end{tabular}

\begin{tabular}{cccccc}
\hline $\begin{array}{c}\text { Número } \\
\text { muestra }\end{array}$ & Peso vials & $\begin{array}{c}\text { Peso neto } \\
\text { muestra }\end{array}$ & Avance péndulo & $\begin{array}{c}\% \text { avance } \\
\text { respecto a la } \\
\text { Pentolita }\end{array}$ & $\begin{array}{c}\text { \% comparado } \\
\text { con Pentolita }\end{array}$ \\
\hline 1A PENDUL & 9,5173 & 11,9329 & $31 \mathrm{~mm}$ & $73,8 \%$ & $-26,2 \%$ \\
\hline 1B PENDUL & 9,4661 & 12,4241 & $35 \mathrm{~mm}$ & $83,33 \%$ & $-16,67 \%$ \\
\hline
\end{tabular}

\begin{tabular}{ccc}
\hline VOD & Peso contenedor & Peso muestra \\
\hline 11,3269 & 102,3648 \\
\hline
\end{tabular}

Fuente: elaboración propia.

\section{Discusión}

Los anteriores resultados permiten ilustrar cómo preparar explosivos, a partir de sistemas de iniciación de mayor energía que el detonador número 8 y otras variables de los explosivos como el diámetro; esto corrobora los valores estimados en energía y parámetros hallados. Entre estos valores, cabe destacar factores de potencia absoluta o relativa, incluida la velocidad de detonación.

Los principales cálculos termodinámicos de entalpía de formación y potencia absoluta, y así la potencia relativa de un explosivo al igual que las propiedades de explosivos como balance de oxígeno, presión de detonación y volumen de gases entre otros, son importantes al realizar una estimación teórica ideal, para poder con estos resultados realizar una formulación real, donde las pruebas realizadas en el laboratorio y las pruebas balísticas se acercan a los resultados estimados.

Los textos analizados sobre explosivos solo muestran fórmulas y cálculos de los parámetros termodinámicos realizados, siempre con el explosivo ANFO; en algunos de ellos, se reportan solo 
resultados de tales parámetros en tablas, sin explicar el desarrollo y la determinación de las características termodinámicas en los explosivos.

La utilidad de la explicación del proceso de fabricación de explosivos como el descrito en el presente artículo es definir la composición química adecuada, la estimación de energía y las características balísticas de un determinado explosivo, con especificaciones militares o comerciales, usando fundamentos de termodinámica para la estimación de energía y la evaluación de sus propiedades balísticas. Lo anterior permite determinar de manera óptima la energía, desempeño y cantidad del explosivo a usar, en relación a otro estimado en una determinada voladura.

Al calcular y comparar los valores teóricos estimados para las mezclas de composición B (véase tabla 5), se encontraron valores estimados muy cercanos para estas composiciones químicas, a excepción del balance total de oxígeno, lo que implica el empleo de otros sistemas de iniciación de mayor energía, diferentes al detonador número 8 . Por lo demás, las composiciones químicas fabricadas de los otros explosivos como Pentolita y PTX-II fueron suficientes su iniciación con detonador número 8 , por ser los valores estimados en balance total de oxígeno más pequeños que las mezclas de composición B.

Así mismo, los valores estimados de energía (potencia absoluta en peso-PAP), en las mezclas de explosivos como composición B, Pentolita y PTX-II, el mejor desempeño en energía, debería ser las mezclas de composición B, no ocurriendo esto, probablemente por el empleo de sistemas de iniciación de baja energía como es el detonador número 8.

\section{Conclusiones}

El cálculo de los parámetros termodinámicos juega un papel importante en la formulación de un explosivo militar, ya que al tener una estimación correcta de los mismos, podemos realizar un material con energía específica, composición efectiva y estable para ser utilizado.

El balance total de oxígeno en los explosivos; probablemente predice la entrega de energía necesaria del detonador para iniciar el explosivo; esto al no encontrarse coherencia de los resultados obtenidos de las composiciones B preparadas con relación a la Pentolita en los ensayos prueba de péndulo, deformación del cilindro (Prueba de HESS) y factor de potencia

El cálculo de las potencias relativas en peso y volumen de explosivos en relación a la Pentolita permite estimar la cantidad de explosivo a utilizar de este material en relación al patrón utilizado.

Al compararse los resultados de las distintas formulaciones de composición B (cálculos de experimentación) podemos seleccionar el explosivo de mayor entrega de energía, como es la mezcla 1, esto por tener mayor potencia absoluta en peso PAP, menor deficiencia de oxígeno, buen factor de potencia (FP) y volumen de gases entre otros con relación a las otras mezclas II y III de composición B.

Al estimarse el factor de potencia en los explosivos, destacamos la importancia de estimar la velocidad de detonación y la energía de explosivo, para optimizar el desempeño de estos materiales en las distintas voladuras.

Los resultados experimentales obtenidos en la prueba de péndulo, prueba de HESS para la Pentolita y PTX-II son coherentes con los factores de potencia estimados teóricamente; siendo 
menor en factor de potencia y deformación del cilindro (prueba de HESS) para el explosivo PTXII en relación a la Pentolita.

Los resultados de prueba de péndulo y de prueba de HESS en los explosivos PTX-II y Pentolita; indican que la energía entregada inicialmente por el detonador número 8, es suficiente, al concordar su coherencia de los resultados obtenidos tanto en factor de potencia, deformación del cilindro (prueba de HESS).

Al compararse los resultados obtenidos en la prueba de péndulo para Pentolita y PTX-II, muestran mayor elongación en resultado en la prueba del péndulo para el PTX-II en relación a la Pentolita; esto probablemente a la menor deficiencia en oxigeno de este material.

\section{Referencias}

1. Caldas, F.J. (1815). Discurso preliminar que leyó el C. [Ciudadano] Coronel Francisco José de Caldas. El día en que dio principio al Curso Militar del Cuerpo de Ingenieros de la República de Antioquia a expensas del mismo Cuerpo. Medellín: en la Imprenta del Gobierno, por el C. [Ciudadano] Manuel María Viller Calderón. Año de 1815. Tercero de la Independencia (investigación fotográfica y producción facsimilar por Capitán (r) César Augusto Castaño Rubiano y Daniel Clavijo. Cf. Sección Reseńas en esta edición). Bogotá: Escuela de Ingenieros, Ejército Nacional de Colombia, Editorial Planeta).

2. Carrillo, W. (2003). Uso de explosivos en demoliciones con voladuras controladas. Bogotá: Revista Ciencias e Ingeniería Neogranadina, $\mathrm{N}^{\circ} 13$. ISSN 0124-8170, pp. 109-115.

3. Department of theArmy Technical Manual (1984). Military Explosives, TM 214-1300-9, Washington D.C. 12 p.

4. Exsa S.A. (2008). Manualpráctico de voladura. Lima: Cuarta edición, $358 \mathrm{p}$.
5. López, E. (1988). Manual de perforación y voladura de roca. Madrid: Instituto Geominero de España, 32 p.

6. Meyer, R.; Kohler, J. \& Homburg, A. (2007). Explosives $\left(6^{\text {th }}\right.$ ed.). Weinheim Alemania: Library of Congress Card No.: Applied for. ISBN 978-3-527-31656-4, 421 p.

7. Paredes Cadena, L. F., dir. (2010). Ingenieros militares en Colombia. 200 años de historia, 1810-2010 (Erwin Rubiano, comp.; Ricardo Esquivel Triana, rev. histórica; César Augusto Castaño Rubiano, Daniel Clavijo, inv. fotográfica). Bogotá: Ejército de Colombia, Escuela de Ingenieros Militares, Editorial Planeta.

8. Sociedad Española de la Dinamita (1928). Estatutos de la Compañia Anónima Explosivos Modernos. Madrid, 35 p.

9. Unión Española de Explosivos, UEE (2002). Manual de empleo de explosivos. Madrid: Imprime graficas Rogar S. A Navalcarnero. 252 p. 